\title{
Expression of Foxp3 and interleukin-7 receptor and clinicopathological characteristics of patients with diffuse large B-cell lymphoma
}

\author{
YAN ZHAO*, WEN-LI CUI* , ZHI-YIN FENG, JING XUE, ABULAJIANG GULINAER and WEI ZHANG
}

\author{
Department of Pathology, The First Affiliated Hospital, Xinjiang Medical University, Xinjiang, Urumqi 830011, P.R. China
}

Received May 15, 2019; Accepted January 8, 2020

DOI: $10.3892 / \mathrm{ol} .2020 .11374$

\begin{abstract}
The most specific biomarker on the surface of regulatory $\mathrm{T}$ cells (Tregs) is the forkhead/wingeded-helix protein 3 (Foxp3). In contrast, the expression of interleukin-7 receptor (IL-7R) is low or negative in Tregs. The present study aimed to investigate the expression of Foxp3 and IL-7R in diffuse large B-cell lymphoma (DLBCL), and to analyse the clinicopathological characteristics of patients with DLBCL and their association with overall survival (OS). Immunohistochemistry was performed to detect the expression of Foxp3 and IL-7R on routinely processed formalin-fixed and paraffin-embedded specimens. The $\chi^{2}$ test was used to analyse the association between the expression of Foxp3 and IL-7R and the clinicopathological characteristics of patients with DLBCL. Survival curves were used to investigate the effect of Foxp3 and IL-7R on patient prognosis. The results demonstrated that high Foxp3 expression in tissue was associated with non- germinal centre B-cell (GCB)-type disease $(\mathrm{P}=0.012)$, International Prognostic Index score $>0 \quad(\mathrm{P}=0.012)$, stage 3 or 4 tumour $(\mathrm{P}=0.045)$ and disease progression and stabilization period $(\mathrm{P}=0.032)$. In addition, $\mathrm{IL}-7 \mathrm{R}$ expression was associated with non-GCB-type disease $(\mathrm{P}=0.001)$ and extranodal lymphoma $(\mathrm{P}=0.008)$. Furthermore, expression of Foxp3 and IL-7R was not associated with OS ( $\mathrm{P}=0.447$ and $\mathrm{P}=0.201$, respectively). Foxp3 and IL-7R expression in non-GCB-type lymphoma was significantly higher compared with that in GCB lymphoma. The expression of Foxp3 and IL-7R may therefore help the development of individualized treatment, prognostic prediction and therapy stratification.
\end{abstract}

Correspondence to: Professor Wei Zhang, Department of Pathology, The First Affiliated Hospital, Xinjiang Medical University, 137 Liyushan South Road, Xinjiang, Urumqi 830011, P.R. China

E-mail: zwyhr100@163.com

*Contributed equally

Key words: diffuse large B-cell lymphoma, forkhead/winged-helix protein 3, interleukin-7 receptor, clinicopathological analysis, immunological targets

\section{Introduction}

Diffuse large B-cell lymphoma (DLBCL) is the most common lymphoid haematopoietic malignancy, accounting for 30-40\% cases of non-Hodgkin's lymphoma (NHL) in developed countries (1). The occurrence of DLBCL is even higher in developing countries. The incidence is generally greater than $40 \%(2,3)$. The age range of patients with DLBCL is relatively wide, and the individual differences regarding the prognosis are clear; the median age is 60-70 years. According to the International Prognostic Index (IPI), those older than 60 years are considered to have poor prognostic factors. DLBCL is a highly invasive disease with a high incidence rate $(4,5)$. DLBCL can be divided into two subgroups, germinal centre B-cell-like (GCB) lymphoma and non-GCB lymphoma, which have direct implications on the prognosis of patients. The 5-year overall survival (OS) rate of the GCB type was $76 \%$, and the 5-year OS rate of the non-GCB type was $34 \%$ (6). The IPI (7) is usually used to predict the prognosis of patients with DLBCL, and has greatly helped clinical decision-making. Changes in the expression of certain genes in the tumour microenvironment will affect the development of tumours and patient prognosis. E.g. CD10, BCL6, IRF4, FOXP1 and GCET1. The IPI presents certain limitations. The IPI score depends on the patient's age, general condition score, clinical stage, number of sites outside the lymph nodes, and whether lactate dehydrogenase is normal. With the development of molecular pathology, gene rearrangements such as MYC have also become a characteristic genetic change of lymphoma. It is therefore crucial to investigate the pathogenesis of DLBCL and identify some prognostic factors of DLBCL.

The transcription factor forkhead/wingeded-helix protein 3 (Foxp3) is the most specific biological marker of regulatory T-cells (Tregs) (8). Foxp3 serves a crucial role in the differentiation of Tregs, which maintain the body's immune homeostasis and possess immunosuppressive functions $(9,10)$. Tregs express high levels of CD4 and CD25 and specifically express Foxp3; however, the expression of interleukin-7 receptor (IL-7R), also known as CD127, in Tregs is low or negative (11). IL-7R is a specific receptor for IL-7. Foxp3 and IL-7R can regulate the expression of downstream target genes by directly binding to the target gene promoter region or synergizing with other transcription factors. Foxp3 and IL-7R participate in multiple biological functions, including 
tumour cell proliferation, apoptosis, invasion, metastasis and peripheral angiogenesis (12).

The purpose of this study was to investigate the prognostic value of Foxp3 and IL-7R protein expression in DLBCL tumour tissues; their association with clinicopathological characteristics, short-term efficacy and long-term survival of patients with DLBCL was determined. The findings from the present study may provide new bases for the clinical treatment decision-making and determination of patients' prognosis.

\section{Materials and methods}

Clinical data. Clinicopathological data were selected from 208 patients with DLBCL, who did not receive any treatment prior to surgery (surgical tumour resection and biopsy) at the Department of Pathology, First Affiliated Hospital of Xinjiang Medical University between January 2005 and December 2012. All patients were diagnosed and classified by two senior pathologists (Dr Wenli Cui and Mr. Zhiyin Feng; Department of Pathology, The First Affiliated Hospital, Xinjiang Medical University, Xinjiang, China) according to the 2008 version of the World Health Organization 'Pathology and Genetics of Hematopoietic and Lymphoid Tissue Neoplasms' (13). The clinicopathological characteristics of patients, including age, sex, tumour site, mass, size, type, clinical stage, IPI, performance status (PS), B symptoms (includes fever, night sweats, and weight loss), extra-ectopic sites, serum lactate dehydrogenase (LDH) level, treatment status, treatment efficiency and follow-up results were collected. The longest follow-up period was 91 months. This study was approved by the Medical Ethics Committee of the First Affiliated Hospital of Xinjiang Medical University. Written informed consent was obtained from each participant prior to biopsy.

Tissue microarray. HE sections of all patients were stored in the hospital. First, the patient cases and pathology numbers were retrieved from the HIS system. The corresponding slices and wax blocks were subsequently collected. The tissues were formalin-fixed and paraffin-embedded (FFPE). One TMA contained sections from 60 patients. All HE sections were analysed by two senior pathologists who were blinded. Two representative regions were selected for each tissue and were marked at the same position in the corresponding wax block. The tissue of the target wax block was punched out vertically by using a Beecher Tissue Arrayer (Beecher Instruments) and embedded in an acceptor wax block to prepare an array containing 120 tissues.

IHC. The EnVision two-step method was used to detect the expression of Foxp3 and IL-7R proteins in tissue microarrays. Samples from the microarray were serially sliced at a thickness of $4 \mu \mathrm{m}$ and mounted on slides. The slides were placed in a baking oven at $37^{\circ} \mathrm{C}$ overnight, dewaxed with xylene (for $20 \mathrm{~min}$, twice), dehydrated with ethanol gradient (concentrations: 100, 100, 95, 80 and 70\%) and washed with tap water and distilled water. The slides were subsequently incubated with $0.3 \% \mathrm{H}_{2} \mathrm{O}_{2}$ for 10 at room temperature to eliminate endogenous peroxidase activity. Antigen retrieval was performed using citrate repair buffer $(\mathrm{pH} 6.0)$ in a $100^{\circ} \mathrm{C}$ oven for $20 \mathrm{~min}$. After cooling, the tissue chips were covered in goat serum (OriGene Technologies, Inc.) and incubated at $37^{\circ} \mathrm{C}$ for $20 \mathrm{~min}$. The sections were incubated with primary antibodies against Foxp3 (cat. no. ab4728; 1:100; Abcam) and IL-7R (cat. no. TA327014; 1:50; OriGene Technologies, Inc.), which were added dropwise according to the manufacturer's protocol, overnight at $4^{\circ} \mathrm{C}$. The tissue chips were washed with PBS and incubated with a secondary antibody that was added dropwise (universal kit, PV-6000, undiluted; OriGene Technologies, Inc.) for 30 at $37^{\circ} \mathrm{C}$. The sections were incubated with 3'-diaminobenzidine (Bei Jing Zhong Shan Jin Qiao) for 5 at $23^{\circ} \mathrm{C}$ to develop the staining. The reaction was stopped using tap water once the slides were stained thoroughly. The slides were counterstained using haematoxylin (14). Gradient ethanol (Qingdao Jisskang Biotechnology Co., Ltd.; 70, 80, 95,100 and $100 \%$ ) was used to rapidly dehydrate the samples, transparent xylene was applied, and neutral resin was used to seal the slide. IHC staining was scored blindly by two independent pathologists based on the proportion of positively stained tumour cells for Foxp3 and IL-7R and the intensity of the staining. The immunoscores ranged between 0 and 3 as follows: i) 0 , no recognizable staining, referred to as negative (-); ii) 1, slight staining, referred to as weak positive (+); iii) 2, moderate staining, referred to as moderate positive $(++)$; and iv) 3, distinct staining, referred to as strong positive $(+++)$ High expression of Foxp3 and IL-7R was defined as moderate positive staining $(++)$ and strong positive staining $(+++)$ for Foxp3 and IL-7R, whereas low expression of Foxp3 and IL-7R was defined as negative (-) and weak positive (+) staining. A light microscope (DM300; Leica Microsystems GmbH; magnifications, x4, x10 and x20) was used.

Statistical analysis. SPSS 17.0 software (SPSS, Inc.) was used for statistical analysis. Clininopathological data were analysed by the $\chi^{2}$ test. Correlation analysis was performed using the Spearman correlation method. The Kaplan-Meier method was used to evaluate the association between Foxp3 and IL-7R expression and other clinicopathological characteristics and patient prognosis. $\mathrm{P}<0.05$ was considered to indicate a statistically significant difference.

\section{Results}

Clinicopathological data. Among the 208 patients with DLBCL, 124 were male and 84 were female (male:female ratio, 1.48:1). The patient age ranged between 34 and 79 years, and the median age was 60 years. There were 170 cases with a tumour diameter $\leq 10 \mathrm{~cm}, 22$ cases with a tumour diameter $>10 \mathrm{~cm}$ and 16 cases without data on tumour diameter. A total of 140 and 68 patients presented with intranodal lymphoma and extranodal lymphoma, respectively. Among the 208 patients, 110 patients were diagnosed with GCB-type lymphoma, 95 had non-GCB-type lymphoma, and 3 harboured deletion mutations. Overall, 125 patients survived, 43 patients died and 40 patients were lost to follow-up.

IHC staining of Foxp3 and $I L-7 R$. The results from IHC demonstrated that Foxp3 was localized in the nucleus (Fig. 1A) and IL-7R was located in the cytoplasm (Fig. 1B). Detection of Foxp3 expression in the DLBCL tissue microarray revealed that scattered positively stained lymphocytes were 

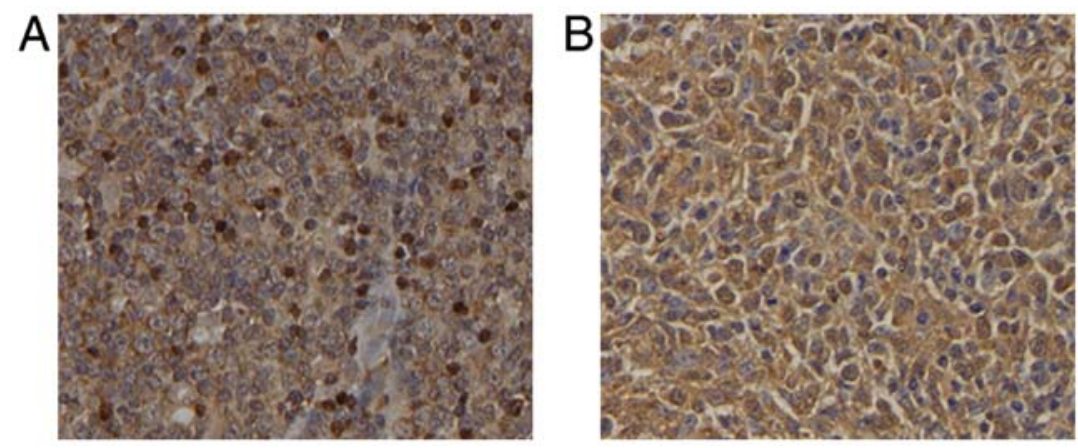

Figure 1. Protein expression at high magnification (x40). (A) Foxp3. (B) IL-7R. Foxp3, forkhead/winged-helix protein 3; IL-R7, interleukin-7 receptor.
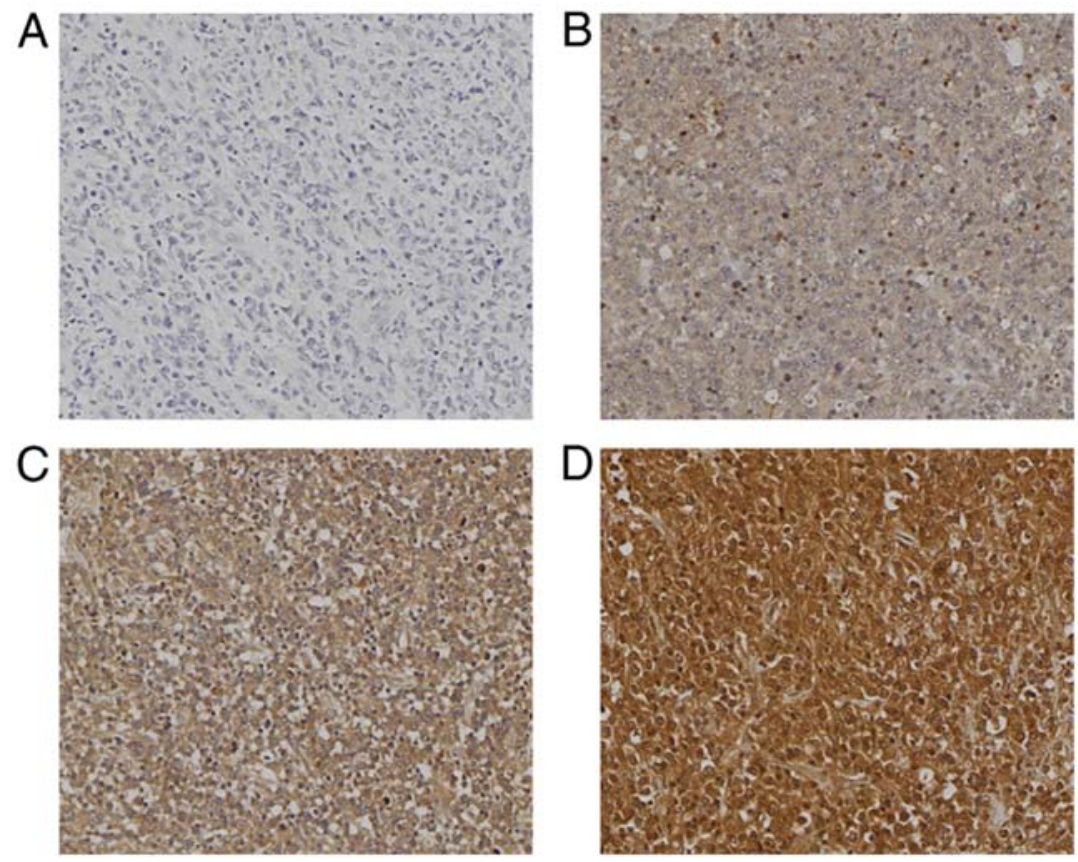

Figure 2. Foxp3 protein is localized in the tumour nucleus. (A) Negative, (B) weakly positive, (C) moderately positive and (D) strongly positive expression of Foxp3 in tissues from patients with diffuse large B-cell lymphoma. Magnification, x20. Foxp3, forkhead/wingeded-helix protein 3.

found both inside and outside of the germinal centre, and presented with different levels of diffuse positive nuclear expression (Fig. 2). The positive staining rate was $65.7 \%$ (132/201 cases). The strongly positive staining rate was $14.4 \%$ (29/201 cases), and high expression was observed in $34.8 \%$ of cases (70/201 cases). Tissue samples from 7 patients were missing. The expression of IL-7R in the DLBCL tissue microarray was detected (Fig. 3). The positive expression rate was 94\% (186/198 cases), strong positive staining was observed in $31.31 \%$ of the cases (62/198 cases), and a high expression rate was observed in $68.2 \%$ of the cases (137/201 cases). Tissue samples from 10 patients were missing.

Association between Foxp3 and IL-7R expression and patient clinicopathological characteristics. To determine the clinicopathological significance of Foxp3 and IL-7R expression, the associations between Foxp3 and IL-7R expression and patient clinicopathological characteristics, including age, size, IPI score, PS, B symptoms and clinical stage, were analysed using $\chi^{2}$ test. No significant association was identified between Foxp3 expression and sex $(\mathrm{P}=0.464)$, age $(\mathrm{P}=0.386)$, tumour size $(\mathrm{P}=0.528)$, tumour site $(\mathrm{P}=0.515)$, $\mathrm{PS}(\mathrm{P}=0.526)$, $\mathrm{B}$ symptoms $(\mathrm{P}=0.347)$, lymph node involvement $>2(\mathrm{P}=0.383)$, LDH serum level $(\mathrm{P}=0.181)$ and treatment plan $(\mathrm{P}=0.218)$. However, a statistically significant association was observed between Foxp3 expression and tumour type ( $\mathrm{P}=0.012)$, IPI score $(\mathrm{P}=0.012)$, tumour stage $(\mathrm{P}=0.045)$ and treatment efficacy $(\mathrm{P}=0.032$; Table I). Furthermore, IL-7R expression was not associated with sex $(\mathrm{P}=0.361)$, age $(\mathrm{P}=0.378)$, tumour size $(\mathrm{P}=0.402)$, IPI score ( $\mathrm{P}=0.099)$, $\mathrm{PS}(\mathrm{P}=0.37)$, $\mathrm{B}$ symptoms $(\mathrm{P}=0.088)$, tumour stage $(\mathrm{P}=0.212)$, lymph node involvement $>2(\mathrm{P}=0.523)$, $\mathrm{LDH}$ serum level $(\mathrm{P}=0.331)$, treatment efficacy $(\mathrm{P}=0.552)$ and treatment regimen $(\mathrm{P}=0.059)$. However, IL-7R expression was associated with tumour type $(\mathrm{P}=0.001)$ and tumour site $(\mathrm{P}=0.008$; Table II). In addition, high Foxp3 expression was associated with non-GCB-type disease, IPI score $>0$, stage 3 and 4 disease, and disease progression and stage $(\mathrm{PD}+\mathrm{SD})($ all $\mathrm{P}<0.05$; Table I) in patients with DLBCL. High expression of IL-7R was also associated with extranodal lymphoma and non-GCB-type disease $(\mathrm{P}<0.05$; Table II). 
Table I. Association between Foxp3 expression and clinicopathological characteristics of patients with diffused large B-cell lymphoma.

\begin{tabular}{|c|c|c|c|c|c|}
\hline \multirow{2}{*}{$\begin{array}{l}\text { Clinicopathological } \\
\text { characteristics }\end{array}$} & \multirow[b]{2}{*}{ Number } & \multicolumn{2}{|c|}{ Foxp3 expression } & \multirow[b]{2}{*}{$\chi^{2}$} & \multirow[b]{2}{*}{ P-value } \\
\hline & & High, $\mathrm{n}$ & Percentage & & \\
\hline \multicolumn{6}{|l|}{ Sex } \\
\hline Male & 120 & 41 & 34.2 & 0.057 & 0.464 \\
\hline Female & 81 & 29 & 35.8 & & \\
\hline \multicolumn{6}{|l|}{ Age, years } \\
\hline$\leq 60$ & 123 & 41 & 33.3 & 0.192 & 0.386 \\
\hline$>60$ & 77 & 28 & 36.4 & & \\
\hline \multicolumn{6}{|l|}{ Diameter, cm } \\
\hline$\leq 10$ & 164 & 57 & 34.8 & 0.022 & 0.528 \\
\hline$>10$ & 22 & 8 & 36.4 & & \\
\hline \multicolumn{6}{|l|}{ Location } \\
\hline Intranodular & 136 & 47 & 34.6 & 0.013 & 0.515 \\
\hline Extranodular & 65 & 23 & 35.4 & & \\
\hline \multicolumn{6}{|l|}{ Subtypes } \\
\hline GCB & 105 & 29 & 27.6 & 5.857 & 0.012 \\
\hline Non-GCB & 93 & 41 & 44.1 & & \\
\hline \multicolumn{6}{|l|}{ IPI } \\
\hline 0 & 104 & 28 & 26.9 & 5.857 & 0.012 \\
\hline$>0$ & 82 & 36 & 43.9 & & \\
\hline \multicolumn{6}{|l|}{ Performance state } \\
\hline$\leq 2$ score & 165 & 59 & 35.8 & 0.022 & 0.526 \\
\hline$>2$ score & 32 & 11 & 34.4 & & \\
\hline \multicolumn{6}{|l|}{ B symptom } \\
\hline No & 99 & 37 & 37.4 & 0.294 & 0.347 \\
\hline Yes & 98 & 33 & 33.7 & & \\
\hline \multicolumn{6}{|l|}{ Clinical stage } \\
\hline 1 and 2 & 112 & 33 & 29.5 & 3.453 & 0.045 \\
\hline 3 and 4 & 75 & 32 & 42.7 & & \\
\hline \multicolumn{6}{|l|}{ Extranodal sites } \\
\hline$\leq 2$ & 158 & 57 & 36.1 & 0.273 & 0.383 \\
\hline$>2$ & 29 & 9 & 31.0 & & \\
\hline \multicolumn{6}{|l|}{$\mathrm{LDH}, \mathrm{U} / 1$} \\
\hline$\leq 240$ & 103 & 32 & 31.1 & 1.141 & 0.181 \\
\hline$>240$ & 83 & 32 & 38.6 & & \\
\hline \multicolumn{6}{|l|}{ Therapeutic effect } \\
\hline $\mathrm{PR}+\mathrm{CR}$ & 90 & 31 & 34.4 & 4.488 & 0.032 \\
\hline $\mathrm{PD}+\mathrm{SD}$ & 20 & 12 & 60.0 & & \\
\hline \multicolumn{6}{|l|}{ R-CHOP treatment } \\
\hline No & 94 & 37 & 39.4 & 0.881 & 0.218 \\
\hline Yes & 80 & 26 & 32.5 & & \\
\hline
\end{tabular}

High expression was defined as moderate positive staining (++) and strong positive staining (+++) for Foxp3. GCB, germinal centre B-cell; Fox3P, forkhead/winged-helix protein 3; IPI, International Prognostic Index; LDH, lactate dehydrogenase; PD, progressive disease; SD, stable disease; PR, partial response; CR, complete response; R-CHOP, rituxan, cyclophosphamide, adriamycin, vincristine, prednisone.

Influence of patient clinicopathological characteristics, Foxp3 and IL-7R expression on prognosis. No significant associations were identified between Foxp3 expression and the overall prognosis of patients using Kaplan-Meier survival curve analysis ( $\mathrm{P}=0.447$; Fig. 4A). IL-7R expression was also not associated with overall prognosis $(\mathrm{P}=0.201$; Fig. 4B). 
Table II. Association between IL-7R expression and clinicopathological characteristics of patients with diffused large B-cell lymphoma.

\begin{tabular}{|c|c|c|c|c|c|}
\hline \multirow{2}{*}{$\begin{array}{l}\text { Clinicopathological } \\
\text { characteristics }\end{array}$} & \multirow[b]{2}{*}{ Number } & \multicolumn{2}{|c|}{ IL-7R expression } & \multirow[b]{2}{*}{$\chi^{2}$} & \multirow[b]{2}{*}{ P-value } \\
\hline & & High, $n$ & Percentage & & \\
\hline \multicolumn{6}{|l|}{ Sex } \\
\hline Male & 118 & 80 & 67.8 & 0.267 & 0.361 \\
\hline Female & 80 & 57 & 71.3 & & \\
\hline \multicolumn{6}{|l|}{ Age, years } \\
\hline$\leq 60$ & 121 & 85 & 70.2 & 0.216 & 0.378 \\
\hline$>60$ & 76 & 51 & 67.1 & & \\
\hline \multicolumn{6}{|l|}{ Size (diameter, $\mathrm{cm}$ ) } \\
\hline$\leq 10$ & 162 & 109 & 67.3 & 0.263 & 0.402 \\
\hline$>10$ & 22 & 16 & 72.7 & & \\
\hline \multicolumn{6}{|l|}{ Location } \\
\hline Intranodular & 134 & 85 & 63.4 & 6.450 & $0.008^{\mathrm{a}}$ \\
\hline Extranodular & 64 & 52 & 81.3 & & \\
\hline \multicolumn{6}{|l|}{ Subtypes } \\
\hline GCB & 103 & 61 & 59.2 & 10.558 & $0.001^{\mathrm{a}}$ \\
\hline Non-GCB & 93 & 75 & 80.6 & & \\
\hline \multicolumn{6}{|l|}{ IPI } \\
\hline 0 & 102 & 64 & 62.7 & 2.088 & 0.099 \\
\hline$>0$ & 81 & 59 & 72.8 & & \\
\hline \multicolumn{6}{|l|}{ Performance state } \\
\hline$\leq 2$ score & 163 & 113 & 69.3 & 0.279 & 0.370 \\
\hline$>2$ score & 31 & 20 & 64.5 & & \\
\hline \multicolumn{6}{|l|}{ B symptom } \\
\hline No & 99 & 63 & 63.6 & 2.271 & 0.088 \\
\hline Yes & 95 & 70 & 73.7 & & \\
\hline \multicolumn{6}{|l|}{ Clinical stage } \\
\hline 1,2 & 111 & 72 & 64.9 & 0.925 & 0.212 \\
\hline 3,4 & 74 & 53 & 71.6 & & \\
\hline \multicolumn{6}{|l|}{ Extra nodal sites } \\
\hline$\leq 2$ & 156 & 105 & 67.3 & 0.031 & 0.523 \\
\hline$>2$ & 29 & 20 & 69.0 & & \\
\hline \multicolumn{6}{|l|}{ LDH, U/1 } \\
\hline$\leq 240$ & 101 & 66 & 65.3 & 0.356 & 0.331 \\
\hline$>240$ & 82 & 57 & 69.5 & & \\
\hline \multicolumn{6}{|l|}{ Therapeutic effect } \\
\hline $\mathrm{PR}+\mathrm{CR}$ & 90 & 57 & 63.3 & 0.020 & 0.552 \\
\hline $\mathrm{PD}+\mathrm{SD}$ & 20 & 13 & 65.0 & & \\
\hline \multicolumn{6}{|l|}{$\mathrm{R}-\mathrm{CHOP}$ treatment } \\
\hline No & 95 & 69 & 72.6 & 2.969 & 0.059 \\
\hline Yes & 78 & 47 & 60.3 & & \\
\hline
\end{tabular}

${ }^{a} \mathrm{P}<0.05$. High expression was defined as moderate positive staining $(++)$ and strong positive staining (+++) for IL-7R. GCB, germinal centre B-cell; IL-7R, interleukin receptor 7; IPI, International Prognostic Index; LDH, lactate dehydrogenase; PD, progressive disease; SD, stable disease; PR, partial response; CR, complete response; R-CHOP, rituxan, cyclophosphamide, adriamycin, vincristine, prednisone.

Subsequently, the association between Foxp3 expression, IL-7R expression and the OS (cum survival) of patients with GCB and non-GCB subtypes was determined; however, the associations were not significant (Fig. 5). Kaplan-Meier survival curve analysis demonstrated that male sex, IPI score $>1$, PS score $>2$, presence of B symptoms, extranodal 

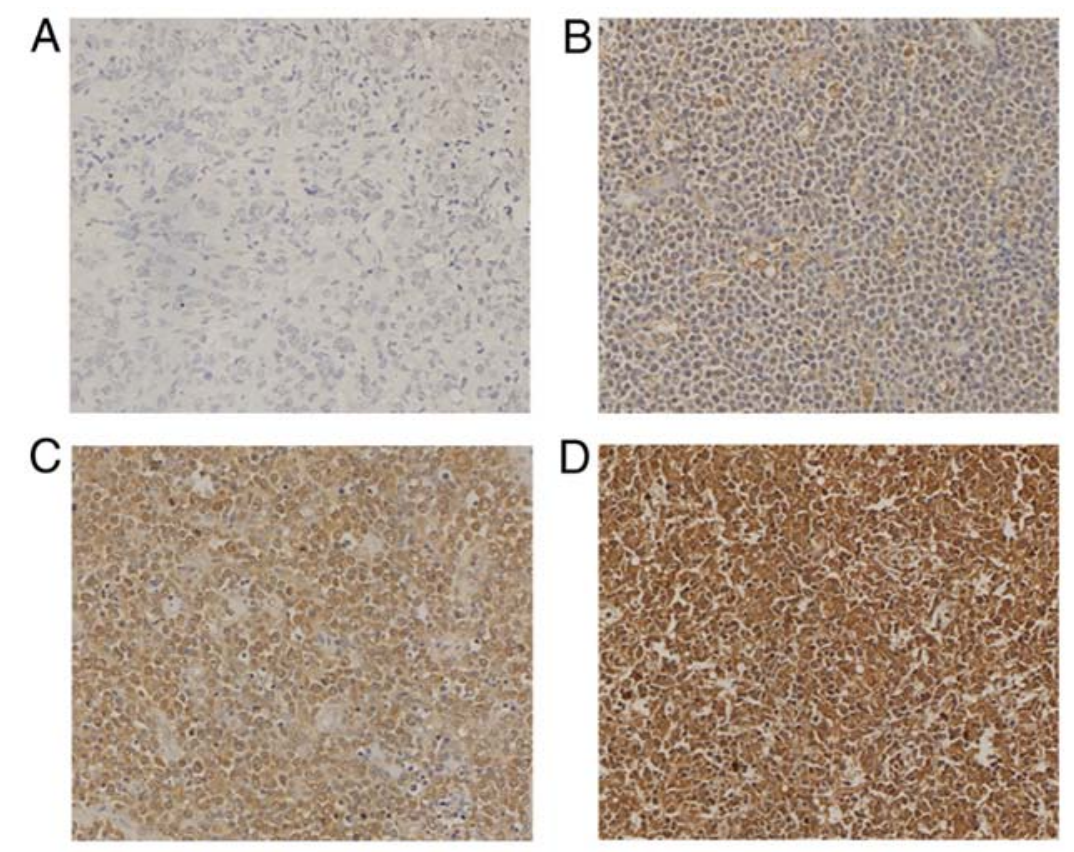

Figure 3. IL-7R protein was located in the tumour cytoplasm. (A) Negative, (B) weakly positive, (C) moderately positive and (D) strongly positive expression of IL-7R in tissues from patients with diffuse large B-cell lymphoma. Magnification, x20. IL-R7, interleukin-7 receptor.

Table III. Spearman correlation analysis of Foxp3 and IL-7R.

\begin{tabular}{lllll}
\hline & \multicolumn{2}{c}{ IL-7R expression } & & \\
\cline { 2 - 3 } Foxp3 expression & Low (\%) & High (\%) & R & P-value \\
\hline Low & $51(25.9)$ & $76(38.6)$ & 0.268 & 0.000 \\
High & $10(5.1)$ & $60(30.5)$ & & \\
\hline
\end{tabular}

Foxp3, forkhead/wingeded-helix protein 3; IL-7R, interleukin-7 receptor.

involvement in $>2$ sites and PS+SD were associated with short survival time (Fig. 4C-H). Spearman correlation analysis demonstrated that expression levels of Foxp3 and IL-7R in patients with DLBCL were weakly positively correlated $(\mathrm{r}=0.268$; Table III).

\section{Discussion}

DLBCL is a lymphoid haematopoietic malignancy. The occurrence and development of DLBCL are closely associated with mutation or loss of regulatory factors (such as PI3K, AKT, P53, MYC, IRF4, BCL-2 and BCL-6) involved in the cell cycle and apoptosis (15). Tumour cell survival depends on the interaction between multiple cells (including tumor cells, T/B lymphocytes and dendritic cells) and their cytokines in the tumour microenvironment $(16,17)$. Foxp3 belongs to the family of forkhead/winged-helix transcription factors and is a characteristic marker located at the surface of Tregs. Foxp3 is located on the X chromosome at Xp11.23. Its gene contains 11 exons and 3 non-coding introns. Foxp3 is associated with immune suppression and immune escape, and its abnormal expression can also lead to autoimmune diseases (such as systemic lupus erythematosus and X-linked autoimmunity-allergic dysregulation syndrome) (18). Previous studies have reported that Foxp3 is expressed by Tregs, as well as in digestive tract tumours, breast cancer, pancreatic cancer and nasopharyngeal carcinoma (19-22). The number of genetic changes in Foxp3 is associated with the occurrence and development of cervical cancer, kidney cancer, lung adenocarcinoma (23), breast cancer (24), colorectal cancer and esophageal cancer (25). However, the expression of Foxp3 in lymphoma remains unclear. Foxp3 has a dual effect, and can upregulate or downregulate multiple oncogenes in tumour cells, including ERBB2, SKP2, c-MYC, p21, and other important cancer-associated genes, leading to transcriptional activation or inhibition (26). Previous studies have reported that elevated numbers of Foxp3-positive cells in the tumour microenvironment indicate a good prognosis for patients with DLBCL. In addition, the number of Foxp3-positive cells in DLBCL is associated with patient prognosis $(25,27,28)$. The results of the present study were similar. Detection of Foxp3 in DLBCL tissue microarrays by IHC demonstrated that Foxp3 was not only expressed in the tumour microenvironment (T lymphocytes) but also presented with diffuse positive expression in DLBCL tumour cells. The results of this study only proved that Foxp3 protein is expressed in the nucleus of DLBCL tumour cells. However, its expression on the surface of Tregs and on normal cells cannot be explained. This study therefore hypothesized that in DLBCL, Foxp3 may be considered as a protein involved in Treg-independent tumour immune escape mechanism. Foxp3 regulates the activation of downstream target genes, which in turn allow tumour cells to evade surveillance by the immune system (29). Foxp3 may serve as a biological marker to predict tumour development and assess prognosis. Wang et al (29) reported that the tumour volume of the Foxp3 high expression group was significantly increased in a subcutaneous tumour-bearing mouse model of pancreatic 

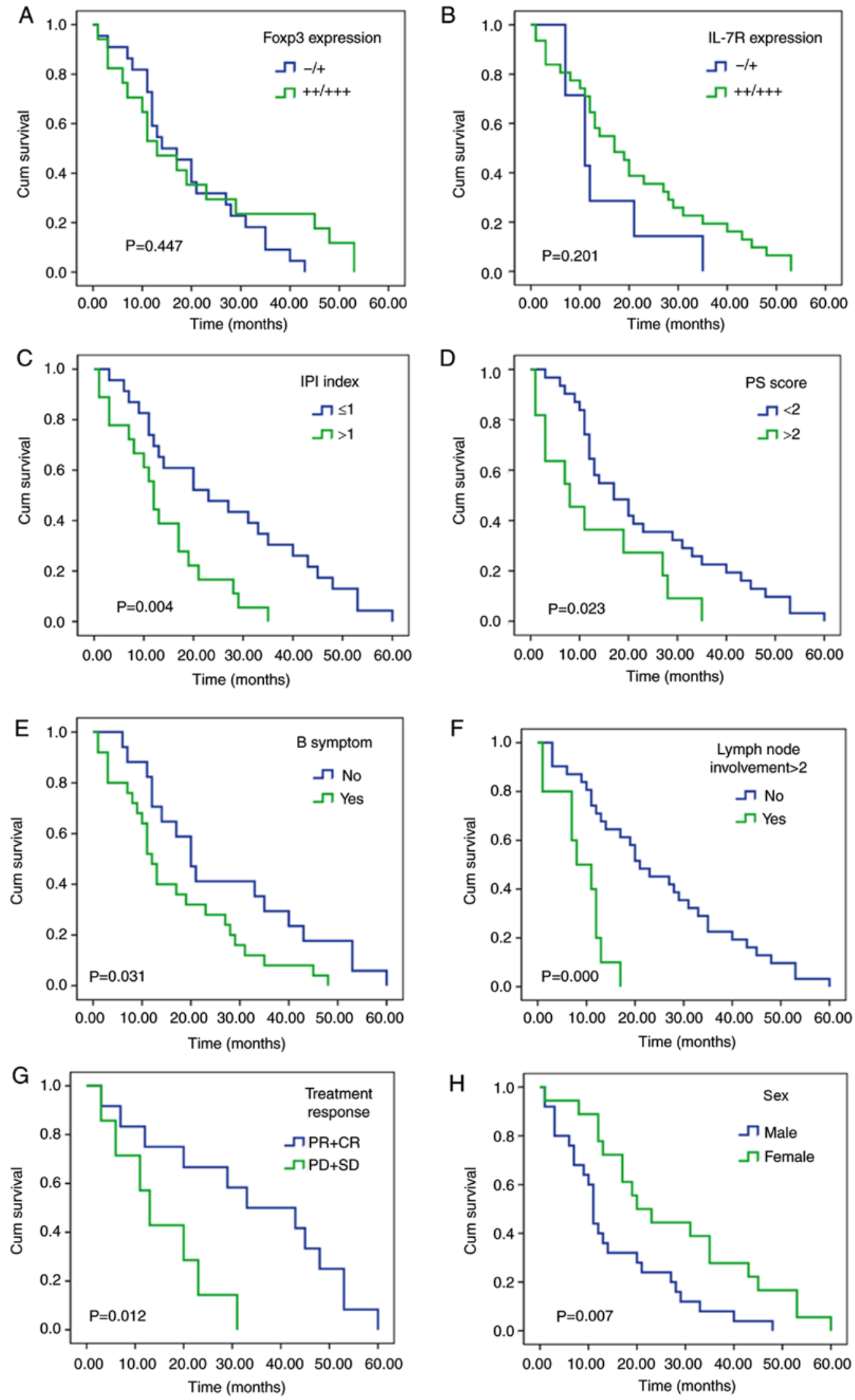

Figure 4. Survival analysis. (A) Survival curves of patients according to Foxp3 expression. (B) Survival curves of patients according to IL-7R expression. (C) Survival curves of patients according to IPI index. Patients with DLBCL and IPI index $>1$ exhibited poor overall survival time. (D) Survival curves of patients according to PS score. Patients with DLBCL and with PS score $>2$ exhibited poor OS. (E) Survival curves of patients according to B symptom. Patients with DLBCL and B symptom exhibited poor OS. (F) Survival curves of patients according to lymph node involvement $>2$. Patients with DLBCL and lymph node involvement $>2$ exhibited poor OS. (G) Survival curves of patients according to the different treatment response. Patients with DLBCL and PD+SD exhibited poor OS. (H) Survival curves of patients according to the sex. Men exhibited poor OS. DLBCL, diffused large B-cell lymphoma; Fox3P, forkhead/winged-helix protein 3; IL-R7, interleukin-7 receptor; IPI, International Prognostic Index; PD, progressive disease; PS, performance status; $\mathrm{SD}$, stable disease; PR, partial response; CR, complete response. 

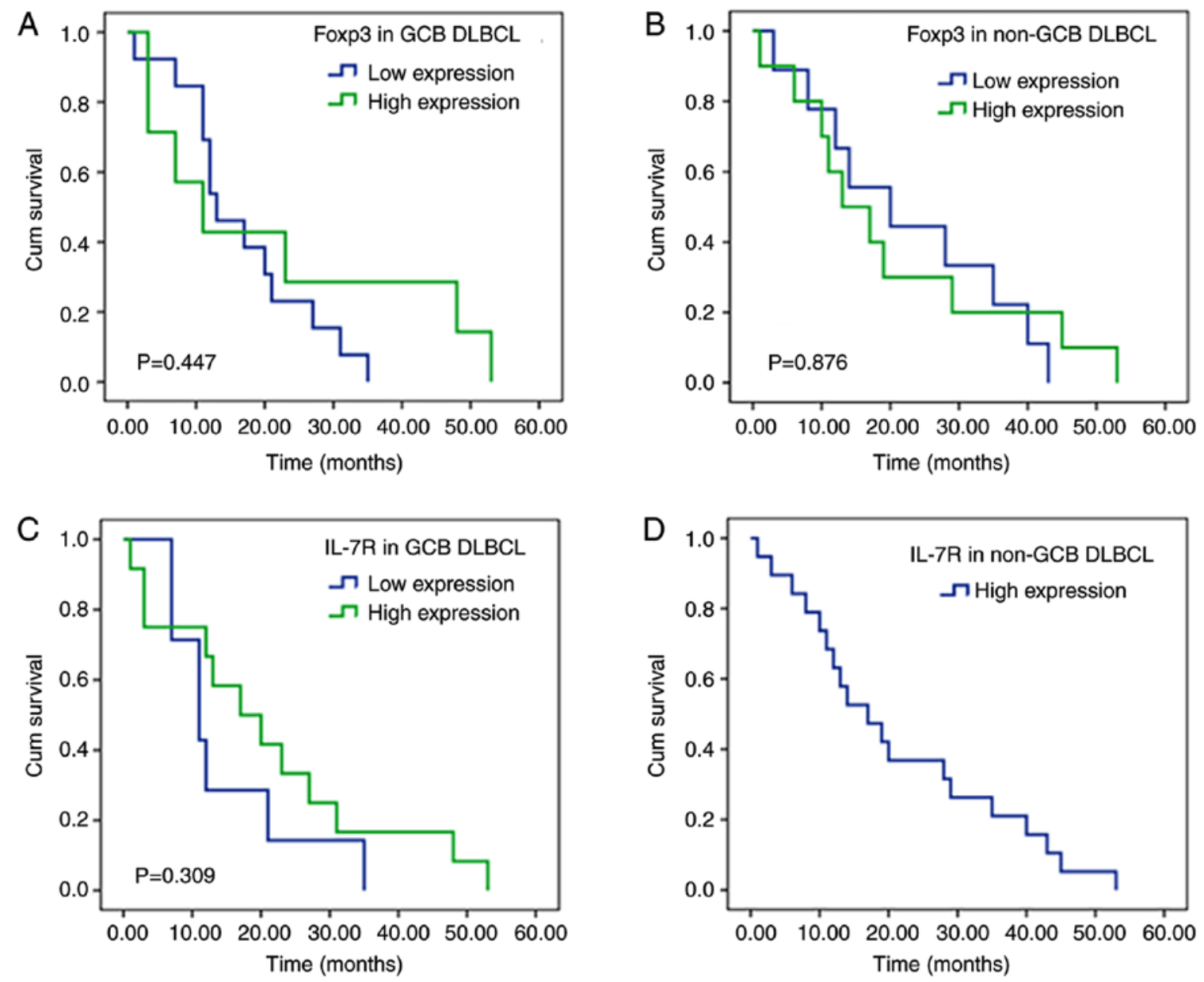

Figure 5. Survival analysis. (A) Survival curves of patients with GCB DLBCL according to Foxp3 expression. (B) Survival curves of patients with non-GCB DLBCL according to Foxp3 expression. (C) Survival curves of patients with GCB DLBCL according to IL-7R expression. (D) Survival curves of patients with non-GCB DLBCL according to IL-7R expression DLBCL, diffused large B-cell lymphoma; Fox3p, forkhead/winged-helix protein 3; GCB, germinal centre B-cell; IL-7R, interleukin-7 receptor.

cancer, and Foxp3 directly transcriptionally activated the expression of the chemokine (C-C motif) ligand 5 (CCL5) in tumour cells and recruited Foxp3-positive Tregs to the tumour microenvironment. These phenomena subsequently inhibited cytotoxic T cell-associated tumour destruction (29). When CCL5 inhibitors were applied, the decreased number of Tregs in the high-Foxp3 expression group was more apparent, and the tumour inhibition rate was higher compared with the low-Foxp3 expression group (29). Furthermore, a previous study reported that Foxp3 can upregulate the expression of transforming growth factor- $\beta$ in tumour cells, leading to the promotion of epithelial-mesenchymal transition and the stimulation of tumour cell proliferation, invasion and metastasis (30).

In the present study, according to the Hans classification model (31), 208 cases of DLBCL were divided into GCB- and non-GCB-type cases. Foxp3 expression was detected by IHC. The results demonstrated that Foxp3 protein was expressed to different degrees in DLBCL tissues, with a positive expression rate of $65.7 \%$ and a strong positive expression rate of $14.4 \%$. Subsequently, the association between Foxp3 expression and clinicopathological characteristics of patients was analysed. High Foxp3 expression was associated with non-GCB-type disease, IPI score $>0$ ), tumour stage 3 or 4 , and PD+SD. These results suggested that Foxp3 expression may be associated with poor prognosis of patients with DLBCL. The results of the survival analysis demonstrated that there was no difference in the survival time between patients with high and low Foxp3 expression levels. Nakayama et al (32) have reported that a high infiltration of FOXP3-positive cells was associated with a significantly better prognosis than patients with low levels of FOXP3-positive cells for OS in DLBCL. In contrast, a high infiltration of FOXP3/CTLA-4 double-positive cells was significantly associated with a poor prognosis compared with patients with low levels of FOXP3/CTLA-4 double-positive cells for OS and progression-free survival. This result is inconsistent with the findings the present study; this may be due to the variable dilution of antibodies. Further investigation is therefore required to determine whether Foxp3 may directly disturb tumour formation or silence genes, affecting the occurrence, development and clinical prognosis of DLBCL.

The IL-7R gene is located on chromosome 5q13 (33). Not only is it a member of the erythropoietin family, but it is also a specific receptor for IL-7. IL-R7 can stimulate haematopoietic cell proliferation and the development of haematological malignancies, including leukaemia and lymphoma $(34,35)$. A study by Sasson et al (36) demonstrated that IL-7R was expressed on human pre-B, but not mature B-cells. Aberrant expression of 
IL-7R contributes to B-cell oncogenesis, which is consistent with the results of the present study. Furthermore, tumour B-cells expressed IL-7R in DLBCL. Al-Rawi et al (37) investigated the expression of IL-7 and IL-7R in solid tumour tissues (breast cancer) and reported that IL-7R expression is positive in cancer tissues and that IL-7R expression is associated with tumour lymph node metastasis. Once IL-7R binds to IL-7, new kinases are required to induce signalling. As the intracellular domain of IL-7R lacks tyrosine kinase activity, signalling is mainly induced through the activation of Jak/STAT, PI3K and the Src family tyrosine kinases (38). The presence of IL-7 abrogated the capacity of $\mathrm{CD}^{+} \mathrm{CD}^{+} 5^{+} \mathrm{Foxp}^{+}$regulatory $\mathrm{T}$ cells (Tregs) to suppress the proliferation of conventional $\mathrm{T}$ cells in response to TCR activators, including alloantigens and autoantigens. The removal of IL-7 restored the suppressive function of Tregs. Preblocking of the IL-7R on the Tregs also restored suppressor function, indicating that IL-7/IL-7R directly affected Tregs function (39). Therefore, the present study investigated the expression of Foxp3 and IL-7R in tumours and their impact on patient OS. To investigate the role of IL-7R in DLBCL, paraffin-embedded samples from 208 patients with DLBCL with complete clinical medical records were examined by IHC. The results demonstrated that IL-7R was highly expressed in DLBCL tissues, which was similar to the results obtained by Al-Rawi et al (37). The present study demonstrated that IL-7R expression was higher in patients with extranodal lymphoma compared with non-extranodal lymphoma $(\mathrm{P}<0.05)$ and higher in patients with non-GCB-type disease compared with those with GCB-type disease $(\mathrm{P}<0.05)$. IL-7R expression may therefore be associated with tumour metastasis. The results of the survival analysis demonstrated no significant differences in the OS between patients with high and low IL-7R expression. In addition, there was no association between IL-7R expression and IPI $>0$ score $(\mathrm{P}=0.099)$, $\mathrm{B}$ symptoms $(\mathrm{P}=0.088)$ or insensitivity to the R-CHOP (40) protocol ( $\mathrm{P}=0.059)$. However, a trend could be detected; when IPI score was high, a B symptom was present, the patient was not sensitive to R-CHOP and IL-7R expression was higher. These results suggested that IL-7R may be involved in the poor prognosis of patients with DLBCL. Further investigation with increased sample size is required to verify these results.

Tregs express high levels of CD4 and CD25, express Foxp3 specifically, and a low level or no IL-7R (11). Thus, IL-7R may also be a specific marker of Tregs (36). The present study demonstrated that Foxp3 and IL-7R was not only expressed on the surface of Treg cells, but also presented with different degrees of positive expression in DLBCL tissues. Furthermore, a weak positive correlation between Foxp3 and IL-7R expression in DLBCL tissues was identified. Foxp3 and IL-7R may represent a tumour immune escape mechanism independent of Tregs. Foxp3 and IL-7R regulate the activation of downstream target genes, leading to the escape of tumour cells from the surveillance of the immune system $(29,38)$. The present study also analysed the association between clinicopathological characteristics of patients with DLBCL and their prognosis. Male sex, IPI index $>1$, PS score $>2$, presence of a B symptom, extranodal involvement in $>2$ sites, and PD+SD were associated with decreased survival time. This study demonstrated that Foxp3 and IL-7R exhibited different degrees of positive expression in DLBCL tissues, and the difference was statistically significant. The establishment of an immunosuppressive microenvironment involving Foxp3 and IL-7R may therefore represent one mechanism involved in DLBCL development. The results from this study may provide novel targets for the development of anti-tumour immunotherapy and help clinicians make appropriate medication choices at an individual level. However, the number of cases was limited. The expression of Foxp3 and IL-7R in DLBCL and the clinicopathological parameters were analysed at an in vitro tissue level. This study lacked the use of in vivo mouse experiments and in vitro cell experiments to confirm the findings.

In conclusion, the expression of Foxp3 and IL-7R in DLBCL tissues was associated with tumour type. In addition, Foxp3 and IL-7R expression in patients with non-GCB-type disease was significantly higher compared with patients with GCB-type disease. The expression of Foxp3 and IL-7R may therefore help the development of individualized treatment, prognostic prediction and therapy stratification.

\section{Acknowledgements}

Not applicable.

\section{Funding}

The present study was supported by the National Natural Science Foundation of China (grant no. NSFC 81560035), the Science and Technology Talents Training Project of Xinjiang Uyghur Autonomous Region (grant nos. qn2015bs011 and 2018Q047) and the Science and Nature Foundation of Xinjiang Uyghur Autonomous Region (grant no. 2014211C032).

\section{Availability of data and materials}

The datasets used and/or analyzed during the current study are available from the corresponding author on reasonable request.

\section{Authors' contributions}

YZ participated in the experiment, analysed the data, and was a major contributor in writing the manuscript. WLC interpreted the results of immunohistochemical experiments. ZYF interpreted the data. JX and AG selected wax blocks and performed immunohistochemistry experiments. WZ designed experimental procedures. All authors read and approved the final manuscript.

\section{Ethics approval and consent to participate}

This study was approved by the Medical Ethics Committee of the First Affiliated Hospital of Xinjiang Medical University (approval no. IACUC-20150225-85). Written informed consent was obtained from all participants.

\section{Patient consent for publication}

Not applicable.

\section{Competing interests}

The authors declare that they have no competing interests. 


\section{References}

1. Herreros B, Sanchez-Aguilera A and Piris MA: Lymphoma microenvironment: Culprit or innocent? Leukemia 22: 49-58, 2008.

2. A clinical evaluation of the International Lymphoma Study Group classification of non-Hodgkin's lymphoma. The Non-Hodgkin's Lymphoma Classification Project. Blood 89: 3909-3918, 1997.

3. Krol AD, le Cessie S, Snijder S, Kluin-Nelemans JC, Kluin PM and Noordijk EM: Primary extranodal non-Hodgkin's lymphoma (NHL): The impact of alternative definitions tested in the Comprehensive cancer centre west population-based NHL registry. Ann Oncol 14: 131-139, 2003.

4. Chiu BC and Hou N: Epidemiology and etiology of non-hodgkin lymphoma. Cancer Treat Res 165: 1-25, 2015.

5. Hamlin PA, Satram-Hoang S, Reyes C, Hoang KQ, Guduru SR and Skettino $S$ : Treatment patterns and comparative effectiveness in elderly diffuse large B-cell lymphoma patients: A surveillance, epidemiology, and end results-medicare analysis. Oncologist 19: $1249-1257,2014$

6. Swerdlow SH, Campo E, Pileri SA, Harris NL, Stein H, Siebert R, Advani R, Ghielmini M, Salles GA, Zelenetz AD and Jaffe ES: The 2016 revision of the World Health Organization classification of lymphoid neoplasms. Blood 127: 2375-2390, 2016.

7. Yim SK, Yhim HY, Han YH, Jeon SY, Lee NR, Song EK, Jeong HJ, Kim HS and Kwak JY: Early risk stratification for diffuse large B-cell lymphoma integrating interim Deauville score and International Prognostic Index. Ann Hematol 98: 2739-2748, 2019.

8. Farinha P, Al-Tourah A, Gill K, Klasa R, Connors JM and Gascoyne RD: The architectural pattern of FOXP3-positive $\mathrm{T}$ cells in follicular lymphoma is an independent predictor of survival and histologic transformation. Blood 115: 289-295, 2010.

9. Mailloux AW and Young MR: Regulatory T-cell trafficking: From thymic development to tumor-induced immune suppression. Crit Rev Immunol 30: 435-447, 2010.

10. Wang K and Vella AT: Regulatory $\mathrm{T}$ cells and cancer: A two-sided story. Immunol Invest 45: 797-812, 2016.

11. Liu W,Putnam AL,Xu-YuZ, Szot GL,Lee MR,Zhu S, GottliebPA, Kapranov P, Gingeras TR, Fazekas de St Groth B, et al: CD127 expression inversely correlates with FoxP3 and suppressive function of human CD4+ Treg cells. J Exp Med 203: 1701-1711, 2006

12. Wang H, Pan K and Xia JC: Interaction of indoleamine-2,3-dioxyagnase and $\mathrm{CD} 4^{+} \mathrm{CD} 25^{+}$regulatory $\mathrm{T}$ cells in tumor immune escape. Ai Zheng 28: 184-187, 2009.

13. Campo E, Swerdlow SH, Harris NL, Pileri S, Stein H and Jaffe ES: The 2008 WHO classification of lymphoid neoplasms and beyond: Evolving concepts and practical applications. Blood 117: 5019-5032, 2011.

14. Cui W, Zheng S, Liu Z, Wang W, Cai Y, Bi R, Cao B and Zhou X: PIK3CA expression in diffuse large B cell lymphoma tissue and the effect of its knockdown in vitro. Onco Targets Ther 10: 2239-2247, 2017.

15. Cui W, Cai Y, Wang W, Liu Z, Wei P, Bi R, Chen W, Sun M and Zhou X: Frequent copy number variations of PI3K/AKT pathway and aberrant protein expressions of PI3K subunits are associated with inferior survival in diffuse large B cell lymphoma. J Transl Med 12: 10, 2014.

16. Keane C, Gould C, Jones K, Hamm D, Talaulikar D, Ellis J, Vari F, Birch S, Han E Wood P, et al: The T-cell receptor repertoire influences the tumor microenvironment and is associated with survival in aggressive B-cell lymphoma. Clin Cancer Res 23: 1820-1828, 2017.

17. Gomez-Gelvez JC, Salama ME, Perkins SL, Leavitt M and Inamdar KV: Prognostic impact of tumor microenvironment in diffuse large b-cell lymphoma uniformly treated with R-chop chemotherapy. Am J Clin Pathol 145: 514-523, 2016.

18. Zhang J, Wei B, Hu H, Liu F, Tu Y, Zhao $M$ and Wu D: Preliminary study on decreasing the expression of FOXP3 with miR-155 to inhibit diffuse large B-cell lymphoma. Oncol Lett 14: 1711-1718, 2017.

19. Hinz S, Pagerols-Raluy L, Oberg HH, Ammerpohl O, Grüssel S, Sipos B, Grützmann R, Pilarsky C, Ungefroren H, Saeger HD, et al: Foxp3 expression in pancreatic carcinoma cells as a novel mechanism of immune evasion in cancer. Cancer Res 67: 8344-8350, 2007.

20. Zuo T, Wang L, Morrison C, Chang X, Zhang H, Li W, Liu Y, Wang Y, Liu X, Chan MW, et al: FOXP3 is an X-linked breast cancer suppressor gene and an important repressor of the HER-2/ErbB2 oncogene. Cell 129: 1275-1286, 2007.
21. Wang LH, Su L and Wang JT: Correlation between elevated FOXP3 expression and increased lymph node metastasis of gastric cancer. Chin Med J (Engl) 123: 3545-3549, 2010.

22. Triulzi T, Tagliabue E, Balsari A and Casalini P: FOXP3 expression in tumor cells and implications for cancer progression. J Cell Physiol 228: 30-35, 2013.

23. Kinoshita F, Takada K, Yamada Y, Oku Y, Kosai K, Ono Y, Tanaka K, Wakasu S, Oba T, Osoegawa A, et al: Combined evaluation of tumor-infiltrating cd $8+$ and foxp $3+$ lymphocytes provides accurate prognosis in stage ia lung adenocarcinoma. Ann Surg Oncol: 26, 2019 (Epub ahead of print).

24. Zhao X, Li Y, Wang X, Wu J, Yuan Y, Lv S and Ren J: Synergistic association of $\mathrm{FOXP}^{+}$tumor infiltrating lymphocytes with CCL20 expressions with poor prognosis of primary breast cancer: A retrospective cohort study. Medicine (Baltimore) 98: e18403, 2019

25. Shang B, Liu Y, Jiang SJ and Liu Y: Prognostic value of tumor-infiltrating FoxP ${ }^{+}$regulatory $\mathrm{T}$ cells in cancers: A systematic review and meta-analysis. Sci Rep 5: 15179, 2015.

26. Katoh H, Zheng P and Liu Y: Signalling through FOXP3 as an X-linked tumor suppressor. Int J Biochem Cell Biol 42: 1784-1787, 2010.

27. Won KY, Kim GY, Kim HK, Choi SIl, Kim SH, Bae GE, Lim JU and Lim SJ: Tumoral FOXP3 expression is associated with favorable clinicopathological variables and good prognosis in gastric adenocarcinoma: The tumor suppressor function of tumoral FOXP3 is related with the P21 expression in gastric adenocarcinoma. Hum Pathol 68: 112-118, 2017.

28. Hu G, Li Z and Wang S: Tumor-infiltrating FoxP3(+) Tregs predict favorable outcome in colorectal cancer patients: A meta-analysis. Oncotarget 8: 75361-75371, 2017.

29. Wang X, Lang M, Zhao T, Feng X, Zheng C, Huang C, Hao J, Dong J, Luo L and Li X: Cancer-FOXP3 directly activated CCL5 to recruit FOXP3(+)Treg cells in pancreatic ductal adenocarcinoma. Oncogene 36: 3048-3058, 2017.

30. Zhang L, Xu J, Zhang X, Zhang Y, Wang L, Huang X and Xu Z: The role of tumoral foxp3 on cell proliferation, migration, and invasion in gastric cancer. Cell Physiol Biochem 42: 1739-1754, 2017.

31. Berglund M, Thunberg U, Amini RM, Book M, Erlanson M, Linderoth J, Dictor M, Jerkeman M, Cavallin-Ståhl E, Sundström C, et al: Evaluation of immunophenotype in diffuse large B-cell lymphoma and its impact on prognosis. Mod Pathol 18: 1113-1120, 2005.

32. Nakayama S, Yokote T, Akioka T, Hiraoka N, Nishiwaki U, Miyoshi T, Iwaki K, Takayama A, Masuda Y, Hatooka J, et al: Infiltration of effector regulatory $\mathrm{T}$ cells predicts poor prognosis of diffuse large B-cell lymphoma, not otherwise specified. Blood Adv 1: 486-493, 2017.

33. Fry TJ and Mackall CL: The many faces of IL-7: From lymphopoiesis to peripheral $\mathrm{T}$ cell maintenance. J Immunol 174: 6571-6576, 2005.

34. Qin JZ, Zhang CL, Kamarashev J, Dummer R, Burg G and Dobbeling U: Interleukin-7 and interleukin-15 regulate the expression of the bcl-2 and c-myb genes in cutaneous T-cell lymphoma cells. Blood 98: 2778-2783, 2001.

35. Takakuwa T, Nomura S, Matsuzuka F, Inoue H and Aozasa K: Expression of interleukin-7 and its receptor in thyroid lymphoma. Lab Invest 80: 1483-1490, 2000.

36. Sasson SC, Smith S, Seddiki N,Zaunders JJ, Bryant A, Koelsch KK, Weatherall C, Munier ML, McGinley C, Yeung J, et al: IL-7 receptor is expressed on adult pre-B-cell acute lymphoblastic leukemia and other B-cell derived neoplasms and correlates with expression of proliferation and survival markers. Cytokine 50: 58-68, 2010.

37. Al-Rawi MA, Mansel RE and Jiang WG: Interleukin-7 (IL-7) and IL-7 receptor (IL-7R) signalling complex in human solid tumours. Histol Histopathol 18: 911-923, 2003.

38. Görgün G, van der Spek J, Cosenza L, Menevse A and Foss F: Altered biological activity associated with C-terminal modifications of IL-7. Cytokine 20: 17-22, 2002.

39. Heninger AK, Theil A, Wilhelm C, Petzold C, Huebel N, Kretschmer K, Bonifacio E and Monti P: IL-7 abrogates suppressive activity of human $\mathrm{CD} 4^{+} \mathrm{CD} 25^{+} \mathrm{FOXP} 3^{+}$regulatory $\mathrm{T}$ cells and allows expansion of alloreactive and autoreactive $\mathrm{T}$ cells. J Immunol 189: 5649-5658, 2012.

40. Coiffier B and Sarkozy C: Diffuse large B-cell lymphoma: R-CHOP failure-what to do? Hematology Am Soc Hematol Educ Program 2016: 366-378, 2016.

This work is licensed under a Creative Commons Attribution-NonCommercial-NoDerivatives 4.0 International (CC BY-NC-ND 4.0) License. 